\title{
Design of a Biomimetic Upper Body for the Humanoid Robot Robota*
}

\author{
Florent Guenter, Lorenzo Roos, Andre Guignard and Aude G. Billard \\ Autonomous Systems Laboratory 3 \\ Ecole Polytechnique Fédérale de Lausanne - EPFL \\ ME A3 454 Station 9 \\ CH-1015 Lausanne \\ \{florent.guenter, aude.billard\}@epfl.ch
}

\begin{abstract}
This paper presents the current prototype of dollshaped humanoid robot Robota. The use of the robot Robota as part of studies with disabled children sets a number of constraints on its design. In particular, it requires that the robot bears a human likeness both in its body features and in the kinematics of its motions. In this paper, we present the design of a 23 degrees of freedom upper body for Robota, including a 3 DOFs spine, two 7 DOFs arm, a 3 DOFs pair of eyes and a 3 DOFs neck.
\end{abstract}

\section{INTRODUCTION}

The Robota project designs a series of biomimetic humanoid robots [1], [2]. Since 1998, Robota has being used as part of studies with autistic children [3], [4]. These studies compare the effect that human-like features may have on the interest that children with autism show in interacting with another agent. Thus, expressing human-like characteristics, both in the robot's body features and in the robot's behaviors, is a key constraint in the design of Robota. We follow the design of commercial dolls to ensure the realism of the aesthetic of the robot, and, take inspiration in the anatomy of the human body to provide the robot with limbs moving according to a humanlike kinematics. These constraints are, however, downrated by the need to provide a sufficiently small and light platform to be easily carried out by a child.

The early prototype of Robota, used in the studies with children, was endowed with only 5 degrees of freedom. This limited importantly the type of interactions games that could be implemented. For the past two years, we have worked on designing a more complex body to extend the type of possible interactions, see Figure 1. In [5], we presented a prototype of a 7 degrees of freedom arm and of a 3 degrees of freedom pair of eyes for an extended version of Robota. In this paper, we present the recent development of an articulated neck and a spine to endow the robot with human-like motions of its torso.

\section{STATE OF THE ART}

Humanoids form a growing body of robotics research; proof is the increasing importance of this conference (IEEE conf. on Humanoids). We consider two classes of humanoid

* This work is supported in part by the Swiss National Science Foundation, through grant no 620-066127 of the SNF Professorships program and by the European Commission Division IST Future and Emerging Technologies, Integrated Project ROBOT-CUB.

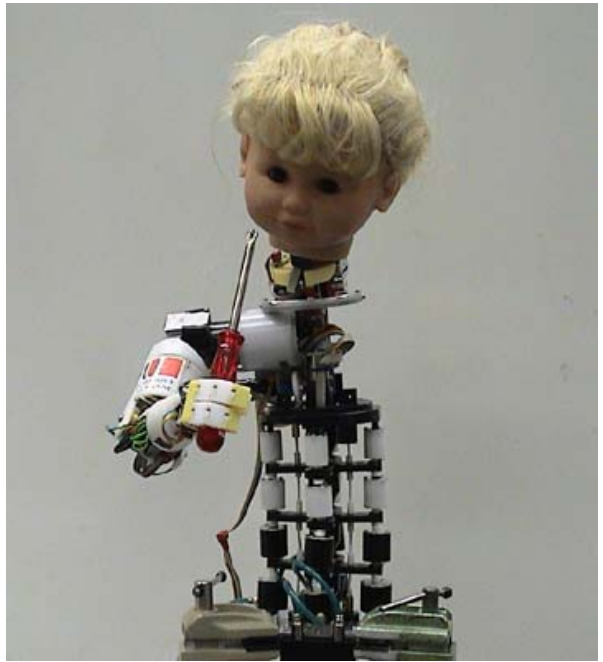

Fig. 1. Current prototype of the new Robota. It encompass a 6 DOFs arm with 1 DOF gripper, 3 DOFs pair of eyes mounted with 2 cameras, 3 DOFs neck and 3 DOFs spinal cord. In a latter stage, the prototype will be embedded in a plastic coating similar to that of commercial doll, , see Figure 3, to ensure that all mechanical parts are hidden, as it is done with the current prototype of Robota, see Figure 2.

robots: those developed by private companies, whose goal is purely commercial, and those developed by universities, whose aims range from modeling how humans control their body to studying the dynamics of human-robot interactions. Among robots developed by private enterprise, we can cite Honda ASIMO [6], Sony Q-RIO [7], Fujitsu HOAP-1\&-2 and HRP-2P [8] developed by the Kawada Industries. Among the robots developed by research groups, we can cite WABIAN [9] from the Waseda university in Japan, CLA and KENTA [10], [11] from the Tokyo university, COG [12] from the MIT or ARMAR [13] from Karlsruhe university ${ }^{1}$.

While these platforms are by far much more advanced than the Robota robot, they, however, do not present a sufficient human-likeliness, as required by the children studies, in which Robota is involved. Note that this needs for human-likeness is

${ }^{1}$ Note that there is a continuum of interactions between these two classes and that robots developed within universities have become commercial products (e.g. HRP, Actroid), whereas commercially produced humanoids are being used by universities (e.g. Fujitsu HOAP-1 and -2, Sony Q-RIO, Sarcos DB). 


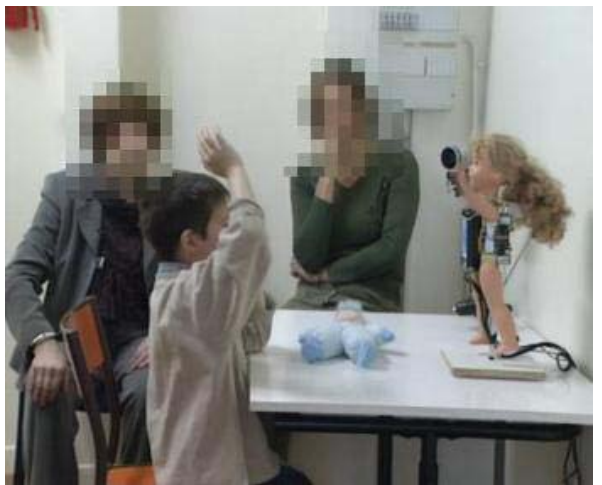

Fig. 2. An autistic child plays a simple imitation game with the robot Robota. The robot detects the motion of the child's hands and head via the camera mounted on top of its Pocket PC and translates these into mirror motion of its corresponding limbs.

not unique to the applications we mentioned and has been stressed by a number of other authors, see, e.g. [14], [15]. At this stage, however, none of these existing robots have humanlike faces (exception being the Replies Q1 and R1 [14] which, however, do not yet produce human-like motions and are of adult-size, too tall for our purpose). Moreover, the majority of existing robots are too large and too heavy, exception being the HOAP and Q-RIO. Unfortunately, the latter robots have been optimized for producing complex locomotion patterns, by increasing the complexity of their lower body to the expense of their upper body. Hence, these robots are not provided with enough degrees of freedom in the upper body to offer enough flexibility for manipulation of objects, a key requirement for most human-robot applications. The Robota projects aims at complementing these approach by providing appealing humanlike robot with an affordable cost ${ }^{2}$.

\section{MECHANICAL DESIGN}

The Robota project is concerned with the design and construction of a series of multiple degrees of freedom (DOF) dollshaped humanoid robots, whose physical features resemble those of a human baby. The Robota project is part of a current trend of robotics research that develops educational and interactive toys for children with disabilities; see, e.g. [16], [17], [15]. As mentioned in introduction, the use of the robot Robota as part of studies with children with autism sets a number of constraints on its design, which we list below:

1) The robot's size and weight must be sufficiently small, so that the child could easily carry it around.

2) The robot's body and facial features must be cute and resemble those of a human baby, so that the robot's effect on the children can be compared to that of a human.

3) The processing must be done on-board for the robot to be easily set-up in the experimental room.

4) The cost of the robot must be sufficiently low for the collaborating schools to be able to purchase it.

${ }^{2}$ Robota is a commercial product sold by DIDEL SA since 1999 at www.didel.com

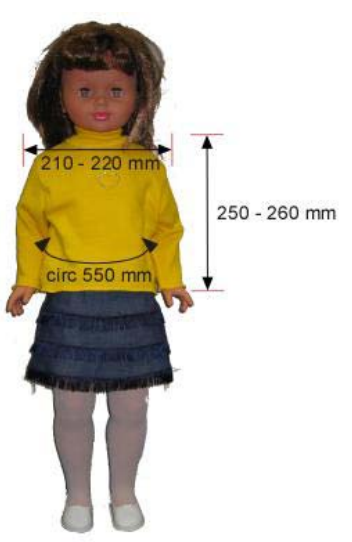

Fig. 3. Doll used as reference for setting the size of the new robot Robota.

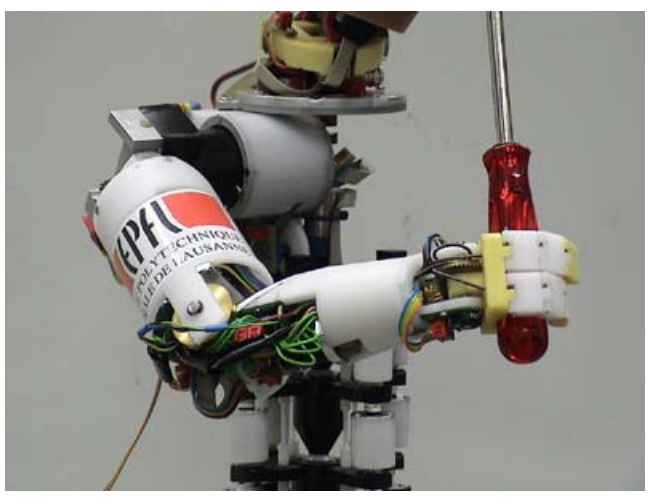

Fig. 4. Prototype of the 7 DOFs arm.

In order to match these constraints, we have designed and built prototypes of a 7 degrees of freedom (DOF) arm, a 3 DOFs pair of eyes, a 3 DOFs neck and a 3 DOFs spine. In order to ensure the overall aesthetic of the construction, we took as reference the average size of a $60 \mathrm{~cm}$ tall commercial doll, see Figure 3, and, the maximal weight of commercial toy robots, i.e. $4 \mathrm{~kg}$. Next, we, first, describe briefly the arm and eyes (a complete technical report of those can be found in [5], [18]). We, then, describe in more details the prototype of neck and spinal cord.

\section{A. Seven degrees of freedom arm}

Figure 4 shows our current prototype of a 6 DOFs arm with a 1 DOF gripper. The arm is 26 centimeter long for $700 \mathrm{gr}$ and a payload of 200 gr. [5]. Each DOF is backdrivable.

In order to obtain human like movements, the different DOFs were placed in the following order ${ }^{3}$ : shoulder flexionextension, shoulder abduction-adduction, shoulder humeral rotation, elbow flexion-extension, wrist rotation, wrist flexionextension and gripper (see Fig. 5). Mechanical stops ensure that each DOF was bounded within the limits of the corresponding DOF of the human arm. Because of cost and space

\footnotetext{
${ }^{3}$ We are aware of the fact that the human arm is not controlled by a serial muscular system. Nevertheless, the order in which the rotation axes have been placed gives the closest equivalent to the human arm motion.
} 


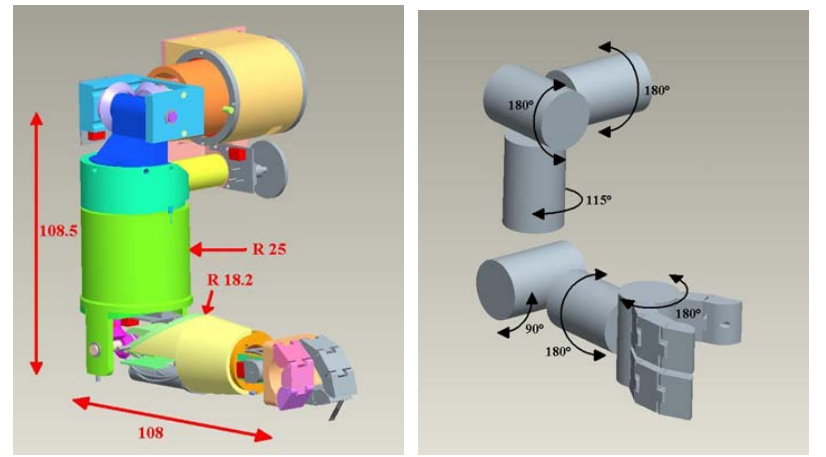

Fig. 5. Left: Dimensions of our 7 DOFs arm (1 DOF in the gripper); Right: Kinematic chain.

constraints, we had to rule out solutions closer to the human control system (e.g. hydraulic motors, linear actuators) and use electric rotative motors with a serial placement of the joints.

The first three degrees of freedom are placed in the shoulder. The three rotation axes cross at the same point (see Figure 5). This implies that the elbow moves on a sphere. We have 1 DOF in the elbow and 2 DOFs in the wrist. The gripper is composed of 3 fingers actuated by a single motor. The fingers are controlled by traction through a set of cables and of opposite springs. This system has the advantage that the fingers adapt to the form of the object they can grasp and can easily be opened by the child (e.g. if the robot should by mistake grasp the child's hand).

In order to obtain an absolute measure of the position of each joint, we have placed potentiometers on each axis. We can, thus, measure the absolute position of the arm when the arm is switched on, and, hence, initialize the motor encoders without having to send the motors to the reset position. This ensures minimal risks when the robot interacts with children, by preventing any involuntary motion if the robot should reset itself.

A major constraint for the design of the arm was to ensure that all the actuators and the electronics would fit in the arm, and, that nothing would protrude. The latter constraint is in striking contrast to the majority of current humanoid arms, where the motor of the 2nd DOF on the shoulder is usually placed on the arm segment and perpendicular to the axis of rotation of the 1st DOF; as a result, the 2 nd DOF protrudes from the arm; a system that is not very aesthetic and may turn out to be dangerous (e.g. pinching a child's hand when rotating backwards).

In addition, we designed the upper and lower arm segments using cylinders with differential diameters, so that the diameters at the shoulder, elbow and wrist would match those of the doll. As a result, the arm appears to respect better the proportions of a human arm.

\section{B. Three degrees of freedom pair of eyes}

Keeping the same constraints as stated in Section III, we have developed a prototype of a 3 DOFs pair of eyes (see Figure 6). One DOF drives the horizontal rotation of the two
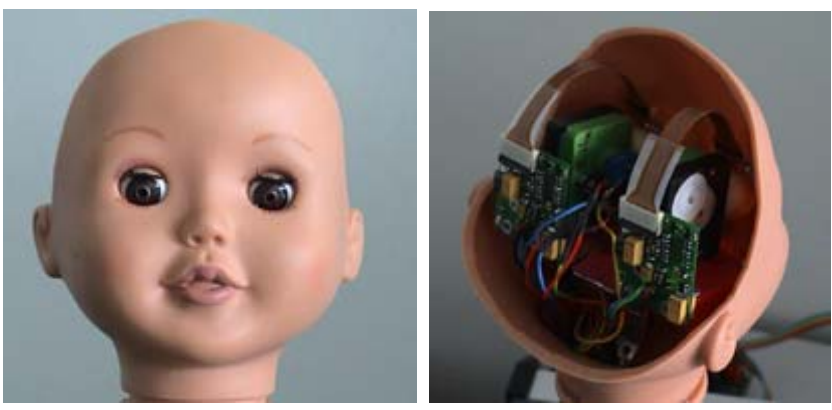

Fig. 6. 3 degrees of freedom pair of eyes for Robota. To ensure that the overall aesthetic of the robot is conserved, we made sure that only visible part of the mechanism would be the eyes.
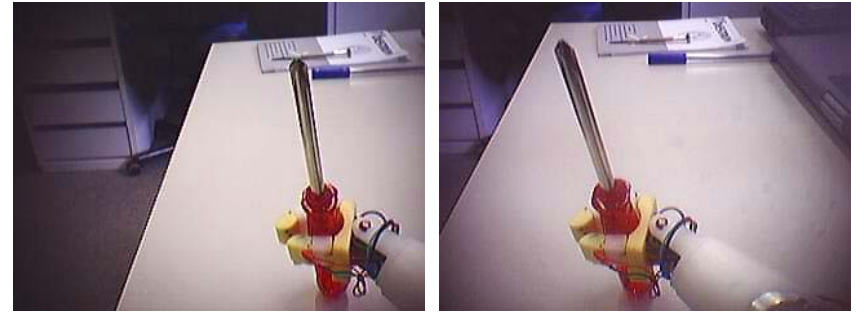

Fig. 7. To enable on board stereovision, we placed in each of Robota's eye a tiny webcam (CMOS 640x480). The two pictures, here, show the images returned by each of Robota's camera when looking down at a screwdriver.

eyes $\left( \pm 45^{\circ}\right)$ and the two other DOFs drive the vertical rotation $\left( \pm 45^{\circ}\right)$ of each eye. Thus, the robot can wink but not squint!

To enable on board stereovision, we placed, in each eye, one "mobile phone" webcam (CMOS 640x480)[18], see Figure 7. We have developed a simple controller for close-loop tracking of human faces, using the feedback of the cameras and a measure of the absolute position of the eyes given by a set of 3 potentiometers. The prototype measures $70 \times 45 \times 50 \mathrm{~mm}$ for a weight of $80 \mathrm{gr}$.

In order to ensure that the overall aesthetic of the face would not be affected by these transformations, we made sure that the only visible part of the mechanism would be the eyes (the rest of the mechanics being hidden inside the doll's head). We used commercially available doll eyes, that we modified to insert the cameras, by drilling a tiny hole through the pupil, making sure that the iris remains intact. We had to finely cut the original doll eyes and mold a complementary part to ensure that the center of rotation of each eye would be located in the middle of the doll eye.

\section{Three degrees of freedom neck}

To design the neck, we followed the same set of constraints as for the other parts of the robot. The number of DOFs is fixed to 3 (lace, pitch and roll). These are placed in series, starting with lace, and, then pitch and roll. The system has been designed to support a load of $400 \mathrm{gr}$, so that it could still drive the head when the robot would be hold upside-down.

Pitch and Roll are controlled by transmission through a set of cables and pulleys, while Lace is controlled by a direct 

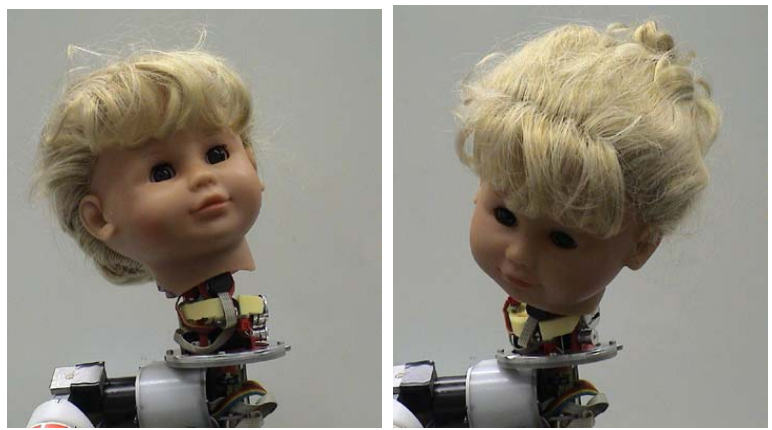

Fig. 8. Realization of the three degrees of freedom neck.

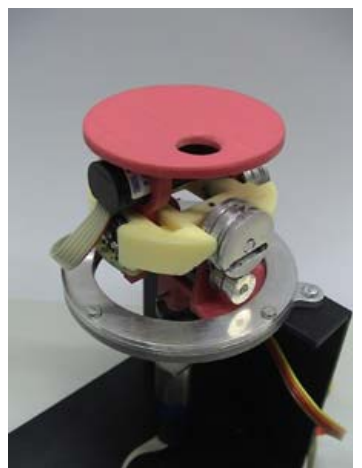

Fig. 9. Prototype of the three degrees of freedom neck.

drive from the motor. Using direct drive and cable transmission minimizes the chance of encountering backlash problems.

Similarly to what was done in the arm, we control the motors in position using a set of built-in encoders. In addition, we have potentiometers to ensure a safe initialization. The current prototype weights $240 \mathrm{gr}$ for $50 \mathrm{~mm}$ height (not including the direct drive motor, see Figure 10) and a mean diameter of $66 \mathrm{~mm}$. The limits of joints are $\pm 80^{\circ}$ for the lace, $\pm 40^{\circ}$ for the pitch and $\pm 30^{\circ}$ for the roll.

\section{Three degrees of freedom chest}

The motivation for designing a spine for our robot were two-fold: first, it would provide the robot with a smoother (human-like) bending of its torso; second, it would be a unique realization of this sort. While there exists a few other prototypes of spines for humanoids robots, see [10], [11], none of these were as small as the one we intended to develop. Ensuring that all components remain small (to fit within the doll's body) while supporting an important load (in proportion to the overall size of each limb) is and has always been a tremendous challenge in all the realizations we have described so far. It was even more so for the creation of the spine, and we had to develop home-made hydraulic actuators.

The current prototype of spine drives two DOFs for frontback and left-right bending respectively, see Figure 12. The third DOF of the torso, supported by the spine, drives the horizontal rotation of the shoulders. The spine is about $200 \mathrm{~mm}$ high for a diameter of $90 \mathrm{~mm}$. It weights about $1 \mathrm{Kg}$ and

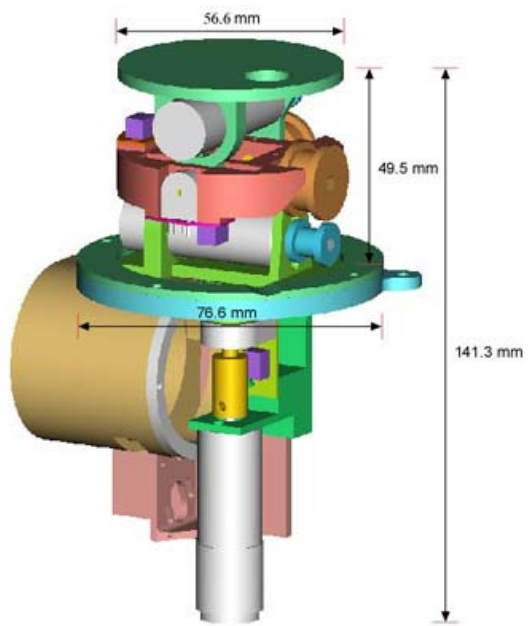

Fig. 10. Dimensions of the neck.

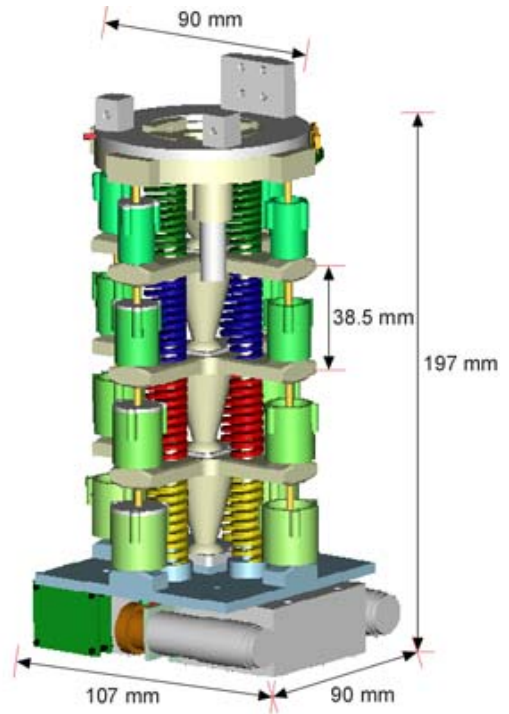

Fig. 11. Dimensions of the torso.
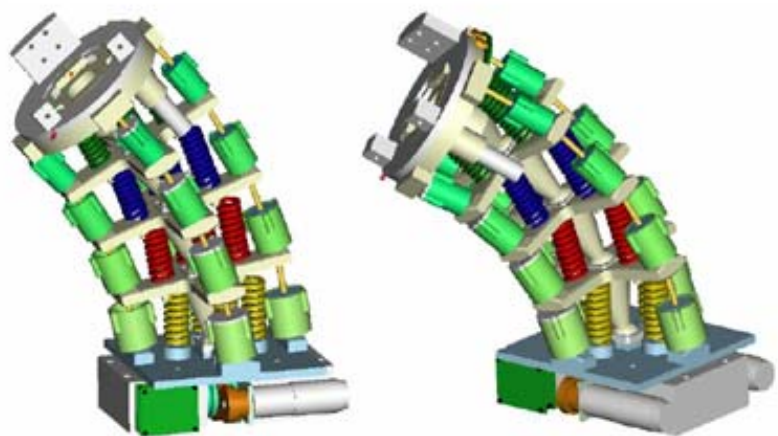

Fig. 12. Maximum bending of the spine. 

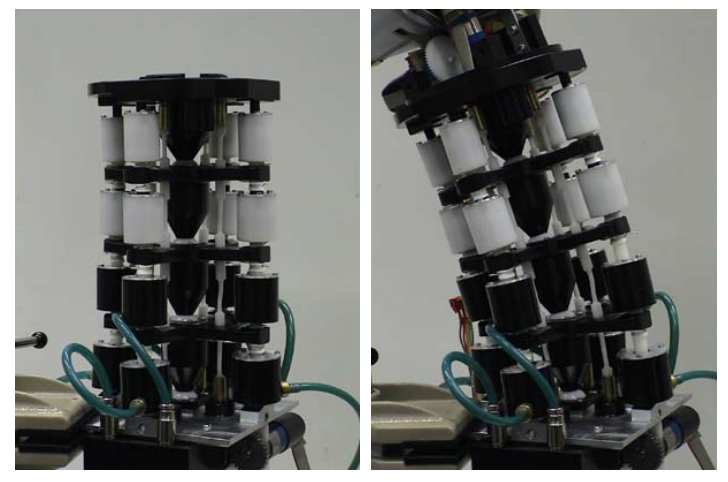

Fig. 13. Current prototype of Robota's spine.

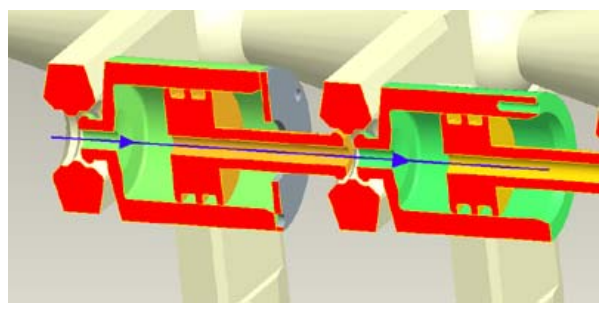

Fig. 14. The oil passes from one piston to another through the piston's axis.

supports a load of $2 \mathrm{Kg}$ located at $80 \mathrm{~mm}$ on the spine. This corresponds to the estimated mass of the current prototype and position of the mass center of the two arms and the head.

To obtain a smooth curvature along the spine, we have used a low pressure hydraulic system. Four pistons are placed at each level of the spine (there are four levels), two for each DOF. To move the spine, we have two motors placed in its base. Using a reduction gear, each motor transmits the movement to an endless screw that moves two pistons working as a pump. Thus, for each DOF there are 2 sets of 4 pistons, one set of pistons are expanding to give the necessary strength to bend the spine while the other retire (without acting on the spine, the pistons work only on compression). Mineral oil is used to transmit the strength. To reduce the number of tubes around the spine, the oil passes from one piston to another trough the piston's axis (see Fig. 14). A rubber tube is used to provide etancheity between two pistons inside vertebra.

Four additional springs were added to each level to increase the support forces and ensure that the spine would not bend nor rotate under the important weight of the head. The springs of lower level are stronger in order to compensate the difference of torque between higher and lower level during bending. The articulations at each level are implemented using spherical bearings.

To control the spine, we have a 2 axes inclinometer placed in the upper vertebra and one in the base of the spine. A differential measurement of the inclination gives us the absolute position of the spine. In order to measure the angle of rotation of the shoulders, we use an incremental encoder, built in the motor.

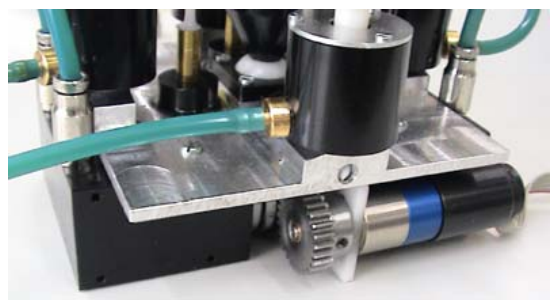

Fig. 15. Prototype of the traction system.

\begin{tabular}{|c|c|c|}
\hline & Neck & Eyes \\
\hline Weight [gr] & 240 & 80 \\
\hline Size $[\mathrm{mm}]$ & $50 \times 66$ & $70 \times 45 \times 50$ \\
\hline Max Torque [nm] & 0.52 & 0.1 \\
\hline Max Speed [Rad/s] & 2.2 & 2.5 \\
\hline Motor control boards & 3 & 3 \\
\hline Motors & 3 Faulhaber 15mm & $\begin{array}{c}\text { 2 Portescap } 6 \mathrm{~mm} \\
\text { 1 Portescap 8mm }\end{array}$ \\
\hline Sensors & $\begin{array}{c}\text { 3 potentiometers } \\
\text { 3 potentiometers }\end{array}$ \\
\hline Cost [\$] & 506 & 262 \\
\hline
\end{tabular}

TABLE I

PRACTICAL DATA ON THE PROTOTYPE OF NECK AND EYES.

\begin{tabular}{|c|c|c|}
\hline & Arm & Spinal Cord \\
\hline Weight [gr] & 700 & 1000 \\
\hline Size [mm] & $260 \times 50$ & $200 \times 90$ \\
\hline Max Torque [nm] & 1.1 & 3 \\
\hline Max Speed [Rad/s] & 2.0 & 1.0 \\
\hline Motor control boards & 7 & 3 \\
\hline Motors & 4 Faulhaber 15mm & 2 Faulhaber 17mm \\
& 1 Faulhaber 22mm & 1 Faulhaber 10mm \\
& 2 Maxon 10mm & \\
\hline Sensors & 6 potentiometers & 2 inclinometers \\
& 7 digital encoders & 3 digital encoders \\
& 2 switches & 2 switches \\
& 2 Hall effect sensors & \\
\hline Cost [\$] & 1390 & 870 \\
\hline
\end{tabular}

TABLE II

PRACTICAL DATA ON THE PROTOTYPE OF ARM AND SPINAL CORD.

\section{ELECTRONIC AND CONTROL}

Each actuators in Robota's upper body is controlled by the same Motor Module that allows control in position, speed and torque. To avoid having too many cables passing trough the articulations, each module is placed as close as possible to the motor it drives. The PC communicates with all the modules via I2C. In total, we have at maximum 6 wires running through any articulation: 2 for the motors power supply, 2 for the logic power supply and 2 for the $\mathrm{I} 2 \mathrm{C}$ communication.

The motor module is basically composed of a microprocessor, a H-bridge amplifier, a current sensor and a quadrature decoder. For each module, the H-bridge and the current sensor must be adapted to the power of the motor it must drive. We use two types of H-bridges, that can supply a current of up to $1 \mathrm{~A}$ and $3 \mathrm{~A}$ respectively. A complete motor module has a surface of $\sim 7 \mathrm{~cm}^{2}$.

Robota's arm is controlled by generating sets of trajectories 
for the hand path and, then, computing the corresponding joint angles by solving the inverse kinematics equation given by: $\overrightarrow{\dot{x}}=\mathbf{J} \overrightarrow{\dot{\theta}}$, where $\mathbf{J}$ is the Jacobian. A solution to this equation can be found using the pseudo-inverse with optimization numerical solution [19].

$$
\overrightarrow{\dot{\theta}}_{c}=\mathbf{J}^{+} \overrightarrow{\dot{x}}+\alpha\left(\mathbf{I}-\mathbf{J}^{+} \mathbf{J}\right) g(\vec{\theta})
$$

Where

$$
\mathbf{J}^{+}=\mathbf{J}^{T}\left(\mathbf{J J}^{T}\right)^{-1}
$$

And

$$
g(\vec{\theta})=\overrightarrow{\theta_{r}}-\vec{\theta}
$$

$\mathbf{J}^{+}$is the Moore-Penrose inverse and $g(\vec{\theta})$ is a optimization term which tends to minimize the distance between the arm position and a rest position, determined as the middle range of each joint angle.

The electronics on board of the robot offers two ways to implement the control of these trajectories. First, we can rely on the PID controllers of each motor module to do a closed-loop control for each final joint position. This, however, may not ensure that the corresponding hand path is correctly reproduced, as some PID controllers may be slower to reach a desired position than others. Second, the trajectory is discretized and the orders are sent continuously to all motors. This ensures that the hand path will be followed closely. However, because the baudrate on the $\mathrm{I} 2 \mathrm{C}$ bus is low, this may result in a very slow motion, especially when running a closed-loop control. So far, we have opted for the first solution, mainly because we are still at the stage where we are testing the different motors. In future work, we will replace the $\mathrm{I} 2 \mathrm{C}$ by a BUS-CAN solution to improve the baudrate.

\section{DISCUSSION AND CONCLUSION}

This paper presented the current prototype of upper body for the robot Robota. The motivation behind the construction of Robota stems from the need to have a robot with realistic human features, and, enough degrees of freedom to perform object manipulation. Current commercially available mini humanoid robots have too few degrees of freedom (especially in the wrist) to perform complex manipulation tasks. Moreover, the majority of these robots have only one DOF in the torso, two DOFs in the neck, five DOFs for each arm and none for the eyes. In Robota, we have more DOFs for each of these parts. The drawback of having so many DOFs is that it requires important torques to drive all of the components. Unfortunately, because we are limited in the space available for the components and cannot afford to design completely new motors (in contrast to large companies such as Honda and Fujitsu), we must rely on commercially available DC motors that are not optimized for such application. In order to produce the required torque, while using sufficiently small motors, we end up using motors that produce a very low speed for each
DOF (much lower than that produced by Q-RIO and HOAP2 ). While this is perfectly suitable for the application of the robot with children (in fact, much safer), it may not be optimal for other applications.

\section{ACKNOWLEDGMENT}

We wish to thank Lorenzo Piccardi and Matteo Calzascia, MSc students at our laboratory, for their contribution in the design of the 7 DOFs arm of Robota.

\section{REFERENCES}

[1] A. Billard, "Play, dreams and imitation in robota," in Socially Intelligent Agents . K. Dautenhahn, B. Edmonds, L. Canamero (eds). Kluwer publisher, 2002.

[2] A. Billard, "Robota: Clever toy and educational tool," Robotics \& Autonomous Systems, vol. 42, pp. 259-269, 2003.

[3] B. Robins, K. Dautenhahn, R. te Boekhorst, and A. Billard, "Effects of repeated exposure of a humanoid robot on children with autism," $S$. Keates, J. Clarkson, P. Langdon and P. Robinson (eds), Designing a More Inclusive World. London Springer-Verlag,pp.225-236, 2004.

[4] K. Dautenhahn and A. Billard, "Games children with autism can play with robota, a humanoid robotics doll," in In Proceedings of the 1st Cambridge Workshop on Universal Access and Assistive Technology, March, 2002

[5] F. Guenter, A. Guignard, L. Piccardi, M. Calzascia, and A. Billard, "Development of a miniature aerticulated arm and pair of eyes for the humanoid robot robota," in IEEE/APS Intl Conference on Mechatronics \& Robotics, 2004.

[6] Y. Sakagami, R. Watanabe, C. Aoyama, S. Matsunaga, and K. Higaki, N. Fujimura, "The intelligent asimo: System overview and integration," in IEEE/RSJ Intl Conference on Intelligent Robots and Systems, 2002.

[7] M. Fujita, Y. Kuroki, T. Ishida, and T. Doi, "A small humanoid robot sdr-4x for entertainment applications," in IEEE/ASME Intl Conference on Advenced Intelligent Mechatronics, 2003.

[8] F. Kanehiro, K. Kaneko, K. Fujiwara, K. Harada, S. Kajita, K. Yokoi, H. Hirukawa, K. Akachi, and T. Isozumi, "The first humanoid robot that has the same size as a human and that can lie down and get up," in IEEE International Conference on Robotics \& Automation, 2003.

[9] J. Yamaguchi, E. Soga, S. Inoue, and A. Takanishi, "Development of a bipedal humanoid robot - control method of whole body cooperative dynamic biped walking," in IEEE International Conference on Robotics \& Automation, 1999.

[10] I. Mizuuchi, M. Inaba, and H. Inoue, "A flexible spine human-form robot - development and control of the posture of the spine," in IEEE/RSJ Intl. Conference on Intelligent Robots and Systems, 2001.

[11] I. Mizuuchi, R. Tajima, T. Yoshikai, D. Sato, K. Nagashima, M. Inaba, Y. Kuniyoshi, and H. Inoue, "The design and control of the flexible spine of a fully tendon-driven humanoid kenta," in IEEE/RSJ Intl. Conference on Intelligent Robots and Systems, 2002.

[12] R. Brooks, C. Breazeal, M. Marjanovic, and M. Scassellati, B. Williamson, "The cog project: Building a humanoid robot," Computation for Metaphors, Analogy, and Agents, LNCS 1562, pp. 52-87, 1999.

[13] T. Asfour, K. Berns, and R. Dillman, "The humanoid robot armar: Design and control," in IEEE/APS Intl Conference on Humanoid Robots, 2000

[14] T. Minato, M. Shimada, H. Ishiguro, and S. Itakura, "Development of an Android Robot for Studying Human-Robot Interaction," in Proc. of the Seventeenth International Conference on Industrial and Engineering Applications of Artificial Intelligence and Expert Systems, 2004.

[15] H. Kozima and H. Yano, "Designing a robot for contingency-detection game," in Working Notes Workshop Robotic \& Virtual Interactive Systems in Autism Therapy, 2001.

[16] C. Plaisant, A. Druin, C. Lathan, K. Dakhan, k. Edwards, J. Vice, and J. Montemayor, "Storytelling robot for pediatric rehabilitation," in ASSETS'O0, 2000.

[17] F. Michaud and C. Thberge-Turmel, "Mobile robotic toys and autism," Socially Intelligent Agents - Creating Relationships with Computers and Robots, Kluwer Academic Publishers, pages 125-132., 2002.

[18] F. Pongas, D. Guenter, A. Guignard, and A. Billard, "Development of a miniature pair of eyes with camera for the humanoid robot robota," in IEEE/APS Intl Conference on Humanoid Robots, 2004.

[19] A. Liegeois, "Automatic supervisory control of the configuration and behavior of multibody mechanisms," IEEE Transactions on Systems, Man, and Cybernetics, vol. 7 (12), pp. 868-871, 1977. 